\title{
Article \\ Numerical comparisons between Bayesian and frequentist low-rank matrix completion: estimation accuracy and uncertainty quantification
}

\author{
The Tien Mai ${ }^{1, *(D)}$
}

1 Oslo Centre for Biostatistics and Epidemiology, Department of Biostatistics, University of Oslo, Norway.; t.t.mai@medisin.uio.no

* Correspondence: t.t.mai@medisin.uio.no

\begin{abstract}
In this paper we perform numerous numerical studies for the problem of low-rank matrix completion. We compare the Bayesian approaches and a recently introduced de-biased estimator which provides a useful way to build confidence intervals of interest. From a theoretical viewpoint, the de-biased estimator comes with a sharp minimax-optimal rate of estimation error whereas the Bayesian approach reaches this rate with an additional logarithmic factor. Our simulation studies show originally interesting results that the de-biased estimator is just as good as the Bayesian estimators. Moreover, Bayesian approaches are much more stable and can outperform the de-biased estimator in the case of small samples. However, we also find that the length of the confidence intervals revealed by the de-biased estimator for an entry is absolutely shorter than the length of the considered credible interval. These suggest further theoretical studies on the estimation error and the concentration for Bayesian methods as they are being quite limited up to present.
\end{abstract}

Keywords: Low-rank matrix; matrix completion; Bayesian method; de-biased estimator; uncertainty quantification; confidence interval.

\section{Introduction}

The goal of low-rank matrix completion is to recover a low-rank matrix from its partially observed entries. This problem has recently received and increased attention due to the emergence of several challenging applications, such as recommender systems (particularly the famous Netflix challenge [5]). Different approaches from frequentist to Bayesian methods have been conducted in this problem, both from theoretical and computational point of views, see for example [1,3,6-9,13,15-17,21,22,24].

From a frequentist point of view, most of the recent methods are usually based on penalized optimization. A seminal result can be found in $[7,8]$ for exact matrix completion (noiseless case) and further developed in the noisy case in $[6,13,20]$. Some efficient algorithms had also been studied, for example see $[11,18,21]$. More particularly, in the notible work [13], the authors studied nuclear-norm penalized estimators and provided reconstruction errors for their methods. Moreover, they also showed that these errors are minimax-optimal (up to a logarithmic factor). Note that the average quadratic error on the entries of a rank- $r$ matrix size $m \times p$ from $n$-observations can not be better than: $r \max (m, p) / n$ [13].

More recently, in a remarkable work [9], de-biased estimators have been proposed for the problem of noisy low-rank matrix completion. The estimation accruracy of this estimator is shown to be sharp in the sense that it reaches the minimax-optimal rate without any additional logarithmic factor. A sharp bound has also been obtained by a different estimator in [12], however its statistical inference is not known up to our knowledge. More importantly, the confident intervals on the reconstruction of entries of 
the underlying matrix are also provided by using the de-biased estimators in the work by [9]. It is noted that conducting uncertainty quantification for matrix completion is not strainghtforward. This is because, in general, the solutions for matrix completion do not admit closed-form and the distributions of the estimates returned by the state-of-the-art algorithms are hard to derive.

On the other hand, uncertainty quantification can be assess normally from a Bayesian perspective that is the unknown matrix is considered as a random variable through a prior distribution and the statistical inference can be made by the posterior distribution, for example the credible intervals. Bayesian methods have been also studied for low-rank matrix completion mainly from a computational viewpoint $[2,4,10,15,16,22$ 24]. Most Bayesian estimators are based on conjugate priors which allow to use Gibb ssampling [2,22] or Variational Bayes methods [16] and these algorithms are fast enough to deal with and actually tested on large datasets like Netflix or MovieLens ${ }^{1}$. However, the theoretical understanding of Bayesian estimators is quite limited, up to ourknowledge, [17] and [3] are the only prominent examples. More specifically, they showed that a Bayesian estimator with a low-rank factorization prior reaches the minimax-optimal rate up to a logarithmic factor and the paper [3] further show that the same rate can be obtained by using a Variational Bayesian estimator.

In this paper, we perform various numerical comparisons on the estimation accuracy (the mean square error, the normalized mean square error and the prediction error, see Section 3) between the de-biased estimator in [9] and the Bayesian methods [3] for which the statistical properties have been well studied thoroughly. Furthermore, we examine in details the behaviour of the confidence intervals revealed by the de-biased estimator against the Bayesian credible intervals. Although Bayesian method has become common in matrix completion, its uncertainty quantification in this problem (e.g. credible interval for recovering entries of the matrix) has receive much more limited attention in the literature.

Results from simulation comparisions release originally interesting messages. More specifically, the de-biased estimator is just as good as the Bayesian estimators on the estimation accuracy, although it is completely successful in improving the ancestry estimator that being de-biased. On the other hand, the Bayesian approaches are much more stable than the de-biased method and in addition they outperform the de-biased estimator especially in case of small samples. However, we find that the $95 \%$ confidence intervals disclosed by the de-biased estimator are significantly sharper in the length than the $89 \%$ and $95 \%$ credible intervals. These evidences suggest that the Bayesian estimators may actually reach the minimax-optimal rate sharply and the log-term could be due to the technical proofs (the PAC-Bayesian bounds technique). Furthermore, the concentration rate of the corresponding Bayesian posterior showed in [3] with a log-term could be sharp.

The rest of the paper is structured as follows. In Section 2 we present the lowrank matrix completion problem, then introduce the de-biased estimator as well as the disclosed confidence interval and provide details on the considered Bayesian estimators. In Section 3, simulation studies comparing the different methods are presented. We discuss and conclude our results in the final section.

\section{Low-rank matrix completion}

\subsection{Model}

Let $M^{*} \in \mathbb{R}^{m \times p}$ be an unknown rank-r matrix of interest. We observe a random subset of noisy entries of $M^{*}$ as

$$
Y_{i j}=M_{i j}^{*}+\mathcal{E}_{i j}, \quad(i, j) \in \Omega
$$

1 http://grouplens.org/datasets/movielens/ 
where $\Omega$ is a subset of indices $\{1, \ldots, m\} \times\{1, \ldots, p\}$ and $\mathcal{E}_{i j} \sim \mathcal{N}\left(0, \sigma^{2}\right)$ are independently generated noise at the location $(i, j)$. We assume that the data are missing uniformly at random. Then, the problem of estimating $M^{*}$ with $n=|\Omega|<m p$ is called the (noisy) low-rank matrix completion problem.

Let $\mathcal{P}_{\Omega}(\cdot): \mathbb{R}^{m \times p} \mapsto \mathbb{R}^{m \times p}$ be the orthogonal projection onto the observed entries in the index set $\Omega$ that

$$
\mathcal{P}_{\Omega}(Y)_{i j}= \begin{cases}Y_{i j}, & \text { if }(i, j) \in \Omega, \\ 0, & \text { if }(i, j) \notin \Omega\end{cases}
$$

Notations:

For a matrix $A \in \mathbb{R}^{m \times p},\|A\|_{F}=\sqrt{\operatorname{trace}\left(A^{\top} A\right)}$ denotes its Frobenius norm and $\|A\|_{*}=\operatorname{trace}\left(\sqrt{A^{\top} A}\right)$ denotes its nuclear norm. $[a \pm b]$ is used to denote the interval $[a-b, a+b]$. We use $I d_{q}$ to denote the identity matrix of dimension $q \times q$.

\subsection{The de-biased estimator}

Let $\hat{M}$ be either the solution of the following nuclear norm regularization [18]

$$
\min _{Z \in \mathbb{R}^{m \times p}} \frac{1}{2}\left\|\mathcal{P}_{\Omega}(Z-Y)\right\|_{F}^{2}+\lambda\|Z\|_{*}
$$

or of the following factorization minimization [11]

$$
\min _{U \in \mathbb{R}^{m \times r}, V \in \mathbb{R}^{p \times r}} \frac{1}{2}\left\|\mathcal{P}_{\Omega}\left(Y-U V^{\top}\right)\right\|_{F}^{2}+\frac{\lambda}{2}\|U\|_{F}^{2}+\frac{\lambda}{2}\|V\|_{F}^{2},
$$

where $\lambda>0$ is a tuning parameter.

Given an estimator $\hat{M}$ as above, the de-biased estimator [9] is defined as

$$
M^{d b}:=\operatorname{Pr}_{\text {rank }-r}\left[\hat{M}-\mathcal{P}_{\Omega}(\hat{M}-Y)\right],
$$

where $\operatorname{Pr}_{\text {rank }-r}(B)=\arg \min _{A: \operatorname{rank}(A) \leq r}\|A-B\|_{F}$ is the projection onto the set of rank-r matrices.

Remark 1. The estimation accuracy of the de-biased estimator, provided in Theorem 3 in [9] under some assumptions, is that $\left\|M^{d b}-M^{*}\right\|_{F}^{2} \leq c \max (n, p) r \sigma^{2} / n$ without any extra logterm and $c$ is universal numerical constant.

\subsubsection{Confidence interval}

Let $\hat{M}=\hat{U} \hat{\Sigma} \hat{V}^{\top}$ be the singular values decomposition of $\hat{M}$. Put

$$
v_{i j}:=\sigma^{2}\left[U_{i .}^{d b}\left(U^{d b \top} U^{d b}\right)^{-1} U_{i .}^{d b \top}+V_{j .}^{d b}\left(V^{d b \top} V^{d b}\right)^{-1} V_{j .}^{d b \top}\right],
$$

where

$$
U^{d b}=\hat{U}\left(\hat{\Sigma}+\lambda I d_{r}\right)^{1 / 2} \text { and } V^{d b}=\hat{V}\left(\hat{\Sigma}+\lambda I d_{r}\right)^{1 / 2} .
$$

Then, given a significance level $\alpha \in(0,1)$, the following interval

$$
\left[M_{i j}^{d b} \pm \Phi^{-1}(1-\alpha / 2) \sqrt{v_{i j}}\right]
$$

is a nearly accurate two-sided $(1-\alpha)$ confidence interval of $M_{i j}^{*}$, where $\Phi(\cdot)$ is the CDF of the standard normal distribution. This is given in Corollary 1 in [9]. 


\subsection{The Bayesian estimators}

The Bayesian estimator studied in [3,17] is given by

$$
M^{B}:=\int M \rho_{\lambda}(M \mid Y) d M
$$

where

$$
\rho_{\lambda}(M \mid Y) \propto L(Y \mid M)^{\lambda} \pi(M)
$$

is the posterior and $L(Y \mid M)^{\lambda}$ is the likelihood raising by $\lambda$. Here $\lambda \in(0,1)$ is a tuning parameter and $\pi(M)$ is the prior distribution.

\subsubsection{Priors}

A popular choice for the priors in Bayesian matrix completion is to assign conditional Gaussian priors to $U \in \mathbb{R}^{m \times K}$ and $V \in \mathbb{R}^{p \times K}$ with

$$
M=U V^{\top}=\sum_{k=1}^{K} U_{. k} V_{. k}^{\top}
$$

for a fixed integer $K \leq \min (m, p)$. More specifically, for $k \in\{1, \ldots, K\}$, independently

$$
\begin{aligned}
U_{. k} & \sim \mathcal{N}\left(0, \gamma_{k} I d_{m}\right), \\
V_{. k} & \sim \mathcal{N}\left(0, \gamma_{k} I d_{p}\right), \\
\gamma_{k}^{-1} & \sim \Gamma(a, b),
\end{aligned}
$$

where $I d_{q}$ is the identity matrix of dimension $q \times q$ and $a, b$ are some tuning parameters. This kind of prior is conjugate for which the conditional posteriors can be derived explicitly in closed form and allows to use the Gibbs sampler, see [22] for details. Some reviews and discussion on low-rank factorization priors can be found in $[1,2]$.

Remark 2. In the case that the rank $r$ is not known, it is natural to take $K$ as large as possible, e.g $K=\min (m, p)$ but this may be computationally prohibitive if $K$ is large.

Remark 3. The estimation error for this Bayesian estimator, under some assumptions, is given in Corollary 4.2 in [3] that $\left\|M^{B}-M^{*}\right\|_{F}^{2} \leq \max (n, p) r \sigma^{2} / n$ with additional log-term by $\log (n \max (m, p))$. It is noted that, in [17], the rate is also reached with additional log-term by $\log (\min (m, p))$ under general sampling distribution however the paper considered some truncations on the prior. prior,

For a given rank-r, we propose to consider the following prior, called fixed-rank-

$$
\begin{aligned}
U_{. k} & \sim \mathcal{N}\left(0, I d_{m}\right), \\
V_{. k} & \sim \mathcal{N}\left(0, I d_{p}\right),
\end{aligned}
$$

for $k=1, \ldots, r$. This prior is a simplified version of the above prior. We note that for $K>r$ the Gibbs sampler of the fixed-rank-prior will be faster than Gibbs sampler for the above prior. However, interestingly, results from simulation for the Bayesian estimator with this prior are slightly better than the one based on the above prior at some point.

Remark 4. We remark that the theoretical estimation error for the Bayesian estimator with the fixed-rank-prior given in (6) remains unchanged following by Corollary 4.2 in [3].

\subsubsection{Credible interval}

Using Bayesian approach, the credibility intervals for the matrix and their functions (e.g. entries) can be easily constructed using the Markov Chain Monte Carlo (MCMC) technique. Here, we focus on the equal-tailed credible interval for an entry. 
More precisely, the credible intervals are reported using the $89 \%$ equal-tailed intervals which is suggested by $[14,19]$ for small posterior samples as in our situations with 500 posterior samples. We noted that to obtain $95 \%$ intervals, an effective posterior sample size of at least 10.000 is recommended [14], which is computationally prohibited to run on all of our simulations. A few examples with 10.000 posterior samples are examined in Figure 1.

\section{Numerical studies}

\subsection{Experimental designs}

In order to access the performance of different estimators, a series of experiments were conducted with simulated data:

- $\quad$ Setting I: In the first setting, we fix $m=100$ and a rank- $r$ matrix $M_{m \times p}^{*}$ is generated as the product of two rank- $r$ matrices,

$$
M^{*}=U_{m \times 2}^{*}\left(V_{p \times 2}^{*}\right)^{\top},
$$

where the entries of $U^{*}$ and $V^{*}$ are i.i.d $\mathcal{N}(0,1)$. With a missing rate $\tau=20 \%, 50 \%$ and $80 \%$, the entries of the matrix $M^{*}$ are observed using a uniform sampling. This sampled set is then corrupted by noise as in (1), where the $\mathcal{E}_{i}$ are i.i.d $\mathcal{N}(0,1)$. We alternate the other dimension by taking $p=100$ and $p=1000$. The rank $r$ is varied between $r=2$ and $r=5$.

- Setting II: The second series of simulations is similar to the first one, except that the matrix $M^{*}$ is no longer rank-r, but it can be well approximated by a rank-r matrix:

$$
M^{*}=U_{m \times r}^{*}\left(V_{m \times r}^{*}\right)^{\top}+\frac{1}{10}\left(A_{m \times 50}\right)\left(B_{m \times 50}\right)^{\top}
$$

where the entries of $A$ and $B$ are i.i.d $\mathcal{N}(0,1)$.

Remark 5. We note that for second series of simulations, with appriximate low-rank matrices, the theory of the de-biased estimator can not be used whereas theoretical guarantees for Bayesian estimators are still validated, see [3,17].

For each setup, we generate 50 data sets (simulation replicates) and report the average and the standard deviation for a measure of error of each estimator over the replicates. The behavior of an estimator (say $\widehat{M}$ ) is evaluated through the mean squared error (MSE) per entry

$$
\text { MSE }:=\frac{1}{m p}\left\|\widehat{M}-M^{*}\right\|_{F}^{2}
$$

and the normalized mean square error (NMSE)

$$
\text { NMSE }:=\frac{\left\|\widehat{M}-M^{*}\right\|_{F}^{2}}{\left\|M^{*}\right\|_{F}^{2}}
$$

We also measure the prediction error by using

$$
\text { Pred }:=\frac{\left\|\mathcal{P}_{\bar{\Omega}}\left(\widehat{M}-M^{*}\right)\right\|_{F}^{2}}{m p-n},
$$

where $\bar{\Omega}$ is the set of un-observed entries.

We compare the de-biased estimator (denoted by 'd.b'), the Bayesian estimator with the fixed-rank-prior (6) (denoted by 'f.Bayes') and the Bayesian estimator with the (flexible rank) prior (5) (denoted by 'Bayes'). As a by-product in calculating the de-biased estimator through the Alternating Least Squares estimator (2), we also report the results for this estimator, denoted it by 'als'. 
The 'als' estimator is available from the R package 'softImpute' [18] and is used with defaulted options. The 'd.b' estimator is run with $\lambda=2.5 \sigma \sqrt{m p}$ as in [9]. The 'f.Bayes' and 'Bayes' estimators are used with tuning parameter $\lambda=1 /\left(4 \sigma^{2}\right)$ and parameters for the prior of 'Bayes' estimator are $K=10, a=1, b=1 / 100$. The Gibbs samplers for these two Bayesian estimators are run with 500 steps and 100 burn-in steps.

\subsection{Results on estimation accuracy}

From the reults in Tables 1 and 2, it is clear to see that the de-biased estimator significantly outperforms its ancestry estimator being de-biased. Whereas, the de-biased estimator is just as good as the Bayesian methods in some case.

More specifically, in Table 1, the de-biased estimator is equally comparative to Bayesian estimators in the case with high rates of observation (say $\tau=20 \%$ or $50 \%$ ). With the case of highly missing rate $\tau=80 \%$, the de-biased estimator returns highly unstable results, this may be because its ancestry estimator (here is the als estimator) is unstable with small observation. However, when the dimension of the matrix increases, the differences between the de-biased estimator and the Bayesian estimators become smaller. This is also recognized for the setting of approximate low-rank matrices as in Table 2 and in Table 3, 4 .

The 'f.Bayes' method returns the best results quite often in terms of all considered errors (MSE, NMSE and Pred) in setting of exact low-rank matrices. However, it is noted that for the setting with true underlying matrix being approximately low-rank, in Table 2 and 4, the 'Bayes' approach is slightly betther than the 'f.Bayes' approach at some point. This can be explained as the 'Bayes' approach employed a kind of approximate low-rank prior through the Gamma prior on the variance of the factor matrices and thus it is able to adapt to the approximate low-rankness.

\subsection{Results on uncertainty quantification}

To examine the uncertainty quantification across the methods, we simulate a matrix as in Section 3.1 then we repeat the observation process 50 times. More precisely, we obtain 50 data sets by replicating the observation of $20 \%, 50 \%$ and $80 \%$ entries of the matrix $M^{*}$ using a uniform sampling and then each sampled set is corrupted by noise as in (1), where the $\mathcal{E}_{i}$ are i.i.d $\mathcal{N}(0,1)$.

Table 3 and 4 report the averaged lengths of the confidence intervals and of the credible intervals as well as the estimation errors of all methods over 50 independent experiments. More precisely, we report the $95 \%$ confidence intervals for the de-biased method. The credible intervals is reported using the $89 \%$ equal-tailed intervals which is suggested by $[14,19]$ for small posterior samples as in our situations with 500 posterior samples. We noted that to obtain $95 \%$ intervals, an effective posterior sample size of at least 10.000 is recommended [14]. A few examples with 10000 posterior samples are given in Figure 1.

A noteworthy conclusion from the results in Table 3 and 4 and Figure 1 is that the lengths of the $95 \%$ confidence intervals revealed by the de-biased method are significantly thinner than the $89 \%$ or $95 \%$ credible intervals. We note that in all of our simulations the confidence intervals always contains the true value of interest and thus we do not report the coverage rate. The credible intervals of the 'f.Bayes' approach are really similar to those based on the 'Bayes' approach.

More particularly, in Figure 1, we compare the limiting Gaussian distribution of the de-biased estimator and posterior samples for the 'f.Bayes' method against the true entries of interest. It is clear that the limiting Gaussian distribution of the de-biased estimator yeilds a sharper tail distribution compare to the distribution of the posterior samples. 


\section{Discussion and Conclusion}

In this paper, we provide extensive numerical comparisions between the de-biased estimator and the Bayesian estimators in the problem of low-rank matrix completion. Results from numerical simulations draw a systematic picture on the behaviour of these estimators originally. More specifically, on the estimation accuracy, the de-biased estimator is just comparable to the Bayesian estimators whereas the Bayesian estimators are much more stable and in some cases can outperform the de-biased estimator especially in the small samples. However, the lengths of the confidence intervals (of the entries) are entirely smaller than the lengths of the credible intervals.

These findings suggest that the considered Bayesian estimators may actually reach the minimax-optimal rate of convergence without additional logarithmic factor. These log-terms could be due to the PAC-Bayesian bounds technique that used to prove the theorical properties of the Bayesian estimator. On the other hand, as shown in [3], the same rate with log-term is proved for the concentration of the corresponding posterior and we conjecture that this rate could not be improved due to the width spreading of credible intervals. These are important questions that remain open up to our knowledge.

Last but not least, it is also important to do the comparisons with the Variational Bayesian (VB) method in [16] where its theoretical guarantees are given in [3], because this method is very popular for matrix completion with large dataset. This will be the objective of our future work. However, we would like to note that, in a preprint [2], the authors had performed some comparisons between the Bayesian approach and the VB method. The message from their works is that we can expect that VB should be more or less as accurate as Bayes, maybe slightly less, but that the credibility intervals would be wrong (see e.g Figure 3 in [2]).

Table 1: Simulation results for Setting I (exact low-rank). The mean and the standard deviation (in parentheses) of each error between the simulation replicates are presented. (MSE: average of mean square error; NMSE: average of normalized mean square error; Pred: average of prediction error; als: the alternating least squares estimator; d.b: the de-biased estimator; f.Bayes: the Bayesian estimator with the fixed-rank-prior; Bayes: the Bayesian estimator with flexible rank prior.)

\begin{tabular}{|c|c|c|c|c|c|c|c|c|}
\hline \multirow[b]{2}{*}{ Errors } & \multicolumn{4}{|c|}{$r=2, p=100, \tau=20 \%$} & \multicolumn{4}{|c|}{$r=5, p=100, \tau=20 \%$} \\
\hline & als & d.b & f.Bayes & Bayes & als & d.b & f.Bayes & Bayes \\
\hline MSE & $0.808(.012)$ & $0.051(.004)$ & $0.051(.003)$ & $0.051(.003)$ & $0.828(.013)$ & $0.130(.005)$ & $0.130(.006)$ & $0.130(.006)$ \\
\hline NMSE & $0.399(.056)$ & $0.025(.004)$ & $0.025(.004)$ & $0.025(.004)$ & $0.167(.014)$ & $0.026(.003)$ & $0.026(.002)$ & $0.026(.003)$ \\
\hline \multirow[t]{3}{*}{ Pred } & $0.054(.004)$ & $0.054(.004)$ & $0.054(.004)$ & $0.054(.004)$ & $0.148(.009)$ & $0.148(.009)$ & $0.148(.009)$ & $0.149(.010)$ \\
\hline & \multicolumn{4}{|c|}{$r=2, p=100, \tau=50 \%$} & \multicolumn{4}{|c|}{$r=5, p=100, \tau=50 \%$} \\
\hline & als & d.b & f.Bayes & Bayes & als & d.b & f.Bayes & Bayes \\
\hline MSE & $0.548(.011)$ & $0.088(.007)$ & $0.088(.007)$ & $0.089(.007)$ & $0.632(.014)$ & $0.233(.012)$ & $0.235(.012)$ & $0.238(.012)$ \\
\hline NMSE & $0.290(.040)$ & $0.046(.007)$ & $0.046(.007)$ & $0.047(.008)$ & $0.130(.014)$ & $0.048(.006)$ & $0.048(.005)$ & $0.049(.006)$ \\
\hline \multirow[t]{3}{*}{ Pred } & $0.093(.007)$ & $0.093(.007)$ & $0.094(.007)$ & $0.095(.008)$ & $0.265(.016)$ & $0.265(.016)$ & $0.268(.016)$ & $0.272(.016)$ \\
\hline & \multicolumn{4}{|c|}{$r=2, p=100, \tau=80 \%$} & \multicolumn{4}{|c|}{$r=5, p=100, \tau=80 \%$} \\
\hline & als & d.b & f.Bayes & Bayes & als & d.b & f.Bayes & Bayes \\
\hline MSE & $0.695(.784)$ & $0.545(.805)$ & $0.286(.027)$ & $0.294(.028)$ & $3.999(.900)$ & $3.755(.912)$ & $1.083(.085)$ & $1.417(.228)$ \\
\hline NMSE & $0.335(.344)$ & $0.261(.354)$ & $0.141(.020)$ & $0.145(.024)$ & $0.813(.177)$ & $0.763(.181)$ & $0.222(.029)$ & $0.289(.051)$ \\
\hline \multirow[t]{3}{*}{ Pred } & $0.618(.979)$ & $0.618(.981)$ & $0.304(.031)$ & $0.313(.033)$ & $4.748(1.123)$ & $4.328(1.116)$ & $1.221(.102)$ & $1.602(.265)$ \\
\hline & \multicolumn{4}{|c|}{$r=2, p=1000, \tau=20 \%$} & \multicolumn{4}{|c|}{$r=5, p=1000, \tau=20 \%$} \\
\hline & als & d.b & f.Bayes & Bayes & als & d.b & f.Bayes & Bayes \\
\hline MSE & $0.806(.004)$ & $0.028(.001)$ & $0.028(.001)$ & $0.028(.001)$ & $0.816(.005)$ & $0.070(.001)$ & $0.070(.001)$ & $0.070(.001)$ \\
\hline NMSE & $0.407(.041)$ & $0.014(.001)$ & $0.014(.001)$ & $0.014(.001)$ & $0.162(.010)$ & $0.013(.001)$ & $0.014(.001)$ & $0.014(.001)$ \\
\hline \multirow[t]{3}{*}{ Pred } & $0.029(.001)$ & $0.029(.001)$ & $0.029(.001)$ & $0.029(.001)$ & $0.075(.002)$ & $0.075(.002)$ & $0.076(.002)$ & $0.076(.002)$ \\
\hline & \multicolumn{4}{|c|}{$r=2, p=1000, \tau=50 \%$} & \multicolumn{4}{|c|}{$r=5, p=1000, \tau=50 \%$} \\
\hline & als & & f.Bayes & Bayes & als & d.b & f.Bayes & Bayes \\
\hline MSE & $0.523(.004)$ & $0.046(.001)$ & $0.046(.001)$ & $0.046(.001)$ & $0.564(.004)$ & $0.119(.002)$ & $0.120(.002)$ & $0.120(.002)$ \\
\hline NMSE & $0.266(.025)$ & $0.023(.002)$ & $0.023(.002)$ & $0.024(.002)$ & $0.115(.007)$ & $0.024(.001)$ & $0.024(.001)$ & $0.024(.001)$ \\
\hline \multirow[t]{3}{*}{ Pred } & $0.048(.002)$ & $0.048(.001)$ & $0.048(.001)$ & $0.048(.001)$ & $0.129(.003)$ & $0.129(.003)$ & $0.129(.003)$ & $0.129(.003)$ \\
\hline & \multicolumn{4}{|c|}{$r=2, p=1000, \tau=80 \%$} & \multicolumn{4}{|c|}{$r=5, p=1000, \tau=80 \%$} \\
\hline & als & d.b & f.Bayes & Bayes & als & d.b & f.Bayes & Bayes \\
\hline MSE & $0.312(.005)$ & $0.133(.004)$ & $0.134(.004)$ & $0.134(.004)$ & $0.580(.156)$ & $0.435(.161)$ & $0.404(.012)$ & $0.406(.012)$ \\
\hline NMSE & $0.174(.089)$ & $0.068(.009)$ & $0.068(.009)$ & $0.069(.009)$ & $0.116(.035)$ & $0.087(.035)$ & $0.081(.006)$ & $0.081(.006)$ \\
\hline Pred & $0.175(.258)$ & $0.139(.005)$ & $0.140(.005)$ & $0.140(.005)$ & $0.475(.195)$ & $0.474(.196)$ & $0.436(.014)$ & $0.438(.014)$ \\
\hline
\end{tabular}


Table 2: Simulation results for Setting II (approximate low-rank). The mean and the standard deviation (in parentheses) of each error between the simulation replicates are presented. (MSE: average of mean square error; NMSE: average of normalized mean square error; Pred: average of prediction error; als: the alternating least squares estimator; d.b: the de-biased estimator; f.Bayes: the Bayesian estimator with the fixed-rank-prior; Bayes: the Bayesian estimator with flexible rank prior.)

\begin{tabular}{|c|c|c|c|c|c|c|c|c|}
\hline \multirow[b]{2}{*}{ Errors } & \multicolumn{4}{|c|}{ approximate rank-2, $p=100, \tau=20 \%$} & \multicolumn{4}{|c|}{ approximate rank-5, $p=100, \tau=20 \%$} \\
\hline & als & & & Bayes & als & & & Bayes \\
\hline MSE & $0.915(.012)$ & $0.539(.017)$ & $0.538(.017)$ & $0.537(.017)$ & $0.944(.014)$ & $0.593(.015)$ & $0.592(.015)$ & $0.592(.015)$ \\
\hline NMSE & $0.373(.047)$ & $0.220(.029)$ & $0.219(.028)$ & $0.218(.028)$ & $0.172(.014)$ & $0.108(.009)$ & $0.108(.009)$ & $0.108(.009)$ \\
\hline \multirow[t]{3}{*}{ Pred } & $0.583(.022)$ & $0.583(.022)$ & $0.579(.022)$ & $0.580(.022)$ & $0.718(.030)$ & $0.718(.030)$ & $0.713(.030)$ & $0.712(.030)$ \\
\hline & \multicolumn{4}{|c|}{ approximate rank-2, $p=100, \tau=50 \%$} & \multicolumn{4}{|c|}{ approximate rank-5, $p=100, \tau=50 \%$} \\
\hline & als & d.b & f.Bayes & Bayes & als & d.b & f.Bayes & Bayes \\
\hline MSE & $0.822(.016)$ & $0.593(.018)$ & $0.588(.016)$ & $0.587(.017)$ & $0.956(.021)$ & $0.760(.023)$ & $0.742(.022)$ & $0.743(.022)$ \\
\hline NMSE & $0.324(.038)$ & $0.234(.028)$ & $0.232(.027)$ & $0.232(.027)$ & $0.173(.015)$ & $0.137(.013)$ & $0.134(.012)$ & $0.134(.012)$ \\
\hline \multirow[t]{3}{*}{ Pred } & $0.642(.024)$ & $0.642(.024)$ & $0.633(.021)$ & $0.633(.022)$ & $0.912(.034)$ & $0.913(.034)$ & $0.882(.033)$ & $0.883(.033)$ \\
\hline & \multicolumn{4}{|c|}{ approximate rank-2, $p=100, \tau=80 \%$} & \multirow{2}{*}{\multicolumn{4}{|c|}{ approximate rank-5, $p=100, \tau=80 \%$}} \\
\hline & als & d.b & f.Bayes & Bayes & als & & & \\
\hline MSE & $1.344(.872)$ & $1.275(.887)$ & $0.842(.035)$ & $0.846(.034)$ & 4.747 (1.044) & $4.603(1.064)$ & $1.724(.084)$ & $1.854(.193)$ \\
\hline NMSE & $0.555(.370)$ & $0.527(.375)$ & $0.346(.036)$ & $0.348(.037)$ & $0.876(.181)$ & $0.849(.184)$ & $0.320(.023)$ & $0.344(.043)$ \\
\hline \multirow[t]{3}{*}{ Pred } & $1.430(1.090)$ & $1.431(1.091)$ & $0.902(.040)$ & $0.906(.039)$ & 5.685 (1.303) & $5.305(1.300)$ & $1.963(.100)$ & $2.109(.223)$ \\
\hline & \multicolumn{4}{|c|}{ approximate rank-2, $p=1000, \tau=20 \%$} & \multicolumn{4}{|c|}{ approximate rank-5, $p=1000, \tau=20 \%$} \\
\hline & als & & f.Bayes & Bayes & als & & f.Bayes & Bayes \\
\hline MSE & $0.909(.004)$ & $0.522(.012)$ & $0.522(.012)$ & $0.501(.009)$ & $0.923(.005)$ & $0.552(.011)$ & $0.552(.010)$ & $0.525(.009)$ \\
\hline NMSE & $0.363(.030)$ & $0.208(.017)$ & $0.208(.017)$ & $0.200(.016)$ & $0.168(.009)$ & $0.101(.006)$ & $0.100(.006)$ & $0.095(.005)$ \\
\hline \multirow[t]{3}{*}{ Pred } & $0.546(.013)$ & $0.546(.013)$ & $0.545(.013)$ & $0.532(.011)$ & $0.615(.013)$ & $0.615(.013)$ & $0.613(.012)$ & $0.594(.011)$ \\
\hline & \multicolumn{4}{|c|}{ approximate rank-2, $p=1000, \tau=50 \%$} & \multicolumn{4}{|c|}{ approximate rank-5, $p=1000, \tau=50 \%$} \\
\hline & als & d.b & f.Bayes & Bayes & als & d.b & f.Bayes & Bayes \\
\hline MSE & $0.785(.005)$ & $0.547(.010)$ & $0.546(.010)$ & $0.545(.010)$ & $0.847(.008)$ & 0.62 & $0.622(.012)$ & $0.619(.012)$ \\
\hline NMSE & $0.316(.025)$ & $0.220(.018)$ & $0.219(.018)$ & $0.219(.017)$ & $0.153(.009)$ & $0.113(.007)$ & $0.112(.007)$ & $0.111(.007)$ \\
\hline \multirow[t]{3}{*}{ Pred } & $0.571(.010)$ & $0.571(.010)$ & $0.569(.010)$ & $0.569(.010)$ & $0.694(.014)$ & $0.694(.014)$ & $0.688(.014)$ & $0.687(.013)$ \\
\hline & \multicolumn{4}{|c|}{ approximate rank-2, $p=1000, \tau=80 \%$} & \multirow{2}{*}{\multicolumn{4}{|c|}{$\begin{array}{c}\text { approximate rank- }-5, p=\underset{\text { f.Bayes }}{1000, \tau}=80 \% \\
\text { d.b }\end{array}$}} \\
\hline & als & & f.Bayes & Bayes & als & & f.Bayes & Bayes \\
\hline MSE & $0.767(.014)$ & $0.678(.015)$ & $0.663(.015)$ & $0.662(.015)$ & $1.140(.018)$ & $1.068(.019)$ & $0.992(.020)$ & $0.992(.020)$ \\
\hline NMSE & $0.307(.030)$ & $0.271(.027)$ & $0.265(.026)$ & $0.265(.026)$ & $0.207(.012)$ & $0.194(.011)$ & $0.180(.010)$ & $0.180(.010)$ \\
\hline Pred & $0.709(.017)$ & $0.708(.017)$ & $0.689(.016)$ & $0.689(.016)$ & $1.175(.023)$ & $1.175(.023)$ & $1.080(.023)$ & $1.080(.023)$ \\
\hline
\end{tabular}

Institutional Review Board Statement: Not applicable

Informed Consent Statement: Not applicable

Data Availability Statement: The R codes and data used in the numerical experiments are available at: https://github.com/tienmt/UQMC .

Acknowledgments: The author would like to thank Pierre Alquier for kindly providing useful feedbacks on a first draft of this paper.

Conflicts of Interest: The authors declare no conflict of interest.The funders had no role in the design of the study; in the collection, analyses, or interpretation of data; in the writing of the manuscript, or in the decision to publish the results. 
Table 3: Simulation results for Setting I on average langths of the confidence intervals and of the credible intervals of the entries as well as the mean square errors (standard deviation is given in parentheses).

\begin{tabular}{c|ccc||ccc}
\hline \hline missing rate & CI db & CI f.Bayes & CI Bayes & MSE db & MSE f.Bayes & MSE Bayes \\
\hline & \multicolumn{7}{c}{$r=2, p=100$} \\
\hline$\tau=20 \%$ & $\mathbf{0 . 8 1 1}(.035)$ & $1.028(.055)$ & $1.040(.086)$ & $0.051(.003)$ & $0.051(.003)$ & $0.051(.003)$ \\
$\tau=50 \%$ & $\mathbf{1 . 5 2 1}(.062)$ & $2.430(.226)$ & $2.456(.266)$ & $0.088(.006)$ & $0.088(.007)$ & $0.089(.008)$ \\
$\tau=80 \%$ & $\mathbf{1 . 2 7 1}(.124)$ & $3.418(.589)$ & $3.432(.579)$ & $0.498(.664)$ & $\mathbf{0 . 2 9 0}(.028)$ & $0.295(.030)$ \\
\hline \multicolumn{7}{|c}{$r=2, p=1000$} \\
\hline$\tau=20 \%$ & $\mathbf{0 . 5 6 7}(.013)$ & $0.708(.045)$ & $0.733(.046)$ & $0.028(.001)$ & $0.028(.001)$ & $0.028(.001)$ \\
$\tau=50 \%$ & $\mathbf{0 . 5 4 2}(.019)$ & $0.860(.092)$ & $0.866(.083)$ & $0.046(.001)$ & $0.046(.001)$ & $0.046(.001)$ \\
$\tau=80 \%$ & $\mathbf{0 . 3 1 0}(.027)$ & $0.821(.160)$ & $0.832(.145)$ & $0.364(.977)$ & $\mathbf{0 . 1 3 7}(.004)$ & $0.137(.005)$ \\
\hline \multicolumn{7}{|c|}{$r=5, p=100$} \\
\hline$\tau=20 \%$ & $\mathbf{1 . 2 1 5}(.043)$ & $2.113(.144)$ & $1.521(.101)$ & $0.128(.006)$ & $0.155(.007)$ & $0.128(.006)$ \\
$\tau=50 \%$ & $\mathbf{1 . 1 2 4}(.081)$ & $1.890(.175)$ & $1.907(.171)$ & $\mathbf{0 . 2 3 4}(.012)$ & $0.239(.012)$ & $0.235(.013)$ \\
$\tau=80 \%$ & $\mathbf{1 . 0 3 2}(.301)$ & $3.285(.515)$ & $2.914(.495)$ & $2.768(1.407)$ & $1.799(.117)$ & $\mathbf{1 . 4 9 0}(.156)$ \\
\hline \multicolumn{7}{|c|}{$r=5, p=1000$} \\
\hline$\tau=20 \%$ & $\mathbf{0 . 8 5 6 ( . 0 1 1 )}$ & $1.525(.079)$ & $1.118(.065)$ & $0.070(.002)$ & $0.077(.002)$ & $0.070(.001)$ \\
$\tau=50 \%$ & $\mathbf{1 . 2 3 1}(.021)$ & $2.763(.248)$ & $2.016(.186)$ & $0.120(.003)$ & $0.148(.003)$ & $0.120(.003)$ \\
$\tau=80 \%$ & $\mathbf{0 . 9 2 7}(.052)$ & $2.748(.533)$ & $2.742(.505)$ & $0.418(.011)$ & $0.418(.011)$ & $\mathbf{0 . 4 1 1}(.011)$ \\
\hline \hline
\end{tabular}

Table 4: Simulation results for Setting II on average langths of the confidence intervals and of the credible intervals of the entries as well as the mean square errors (standard deviation is given in parentheses).

\begin{tabular}{c|ccc||ccc}
\hline \hline missing rate & CI db & CI f.Bayes & CI Bayes & MSE db & MSE f.Bayes & MSE Bayes \\
\hline & \multicolumn{5}{c}{ approximate rank-2, $p=100$} \\
\hline$\tau=20 \%$ & $\mathbf{0 . 5 3 0}(.041)$ & $0.691(.070)$ & $0.707(.068)$ & $0.554(.004)$ & $0.553(.004)$ & $\mathbf{0 . 5 5 2}(.004)$ \\
$\tau=50 \%$ & $\mathbf{1 . 2 2 0}(.074)$ & $2.009(.206)$ & $2.023(.191)$ & $0.575(.009)$ & $0.569(.008)$ & $0.569(.008)$ \\
$\tau=80 \%$ & $\mathbf{0 . 8 5 9 ( . 1 4 2 )}$ & $2.338(.496)$ & $2.359(.499)$ & $1.116(.685)$ & $\mathbf{0 . 8 3 0}(.029)$ & $0.835(.030)$ \\
\hline \multicolumn{7}{c}{ approximate rank-2, $p=1000$} \\
\hline$\tau=20 \%$ & $\mathbf{0 . 8 3 1}(.015)$ & $1.054(.065)$ & $1.100(.078)$ & $0.502(.001)$ & $0.501(.001)$ & $\mathbf{0 . 4 8 9}(.005)$ \\
$\tau=50 \%$ & $\mathbf{0 . 5 0 2}(.022)$ & $0.814(.083)$ & $0.824(.083)$ & $0.559(.002)$ & $0.558(.002)$ & $\mathbf{0 . 5 5 7}(.002)$ \\
$\tau=80 \%$ & $\mathbf{0 . 1 8 3}(.022)$ & $0.519(.117)$ & $0.560(.113)$ & $0.673(.006)$ & $0.659(.006)$ & $0.659(.006)$ \\
\hline \multicolumn{7}{|c}{ approximate rank-5, $p=100$} \\
\hline$\tau=20 \%$ & $\mathbf{1 . 1 4 3}(.039)$ & $2.006(.103)$ & $1.462(.084)$ & $0.617(.007)$ & $0.641(.009)$ & $\mathbf{0 . 6 1 4}(.008)$ \\
$\tau=50 \%$ & $\mathbf{1 . 2 4 4}(.070)$ & $2.790(.268)$ & $2.070(.243)$ & $0.749(.019)$ & $0.812(.020)$ & $\mathbf{0 . 7 3 5}(.017)$ \\
$\tau=80 \%$ & $\mathbf{1 . 7 7 1}(.503)$ & $5.461(1.137)$ & $5.415(1.115)$ & $4.946(.819)$ & $2.434(.129)$ & $\mathbf{1 . 9 8 4}(.269)$ \\
\hline \multicolumn{7}{|c|}{ approximate rank-5, $p=1000$} \\
\hline$\tau=20 \%$ & $\mathbf{0 . 9 7 6}(.015)$ & $1.759(.107)$ & $1.338(.090)$ & $0.539(.001)$ & $0.547(.002)$ & $\mathbf{0 . 5 1 8}(.006)$ \\
$\tau=50 \%$ & $\mathbf{0 . 6 8 4}(.015)$ & $1.555(.126)$ & $1.140(.096)$ & $0.619(.003)$ & $0.636(.003)$ & $\mathbf{0 . 6 1 4}(.004)$ \\
$\tau=80 \%$ & $\mathbf{0 . 9 1 5}(.035)$ & $3.312(.563)$ & $2.590(.635)$ & $1.098(.207)$ & $1.156(.016)$ & $\mathbf{0 . 9 9 5}(.012)$ \\
\hline \hline
\end{tabular}



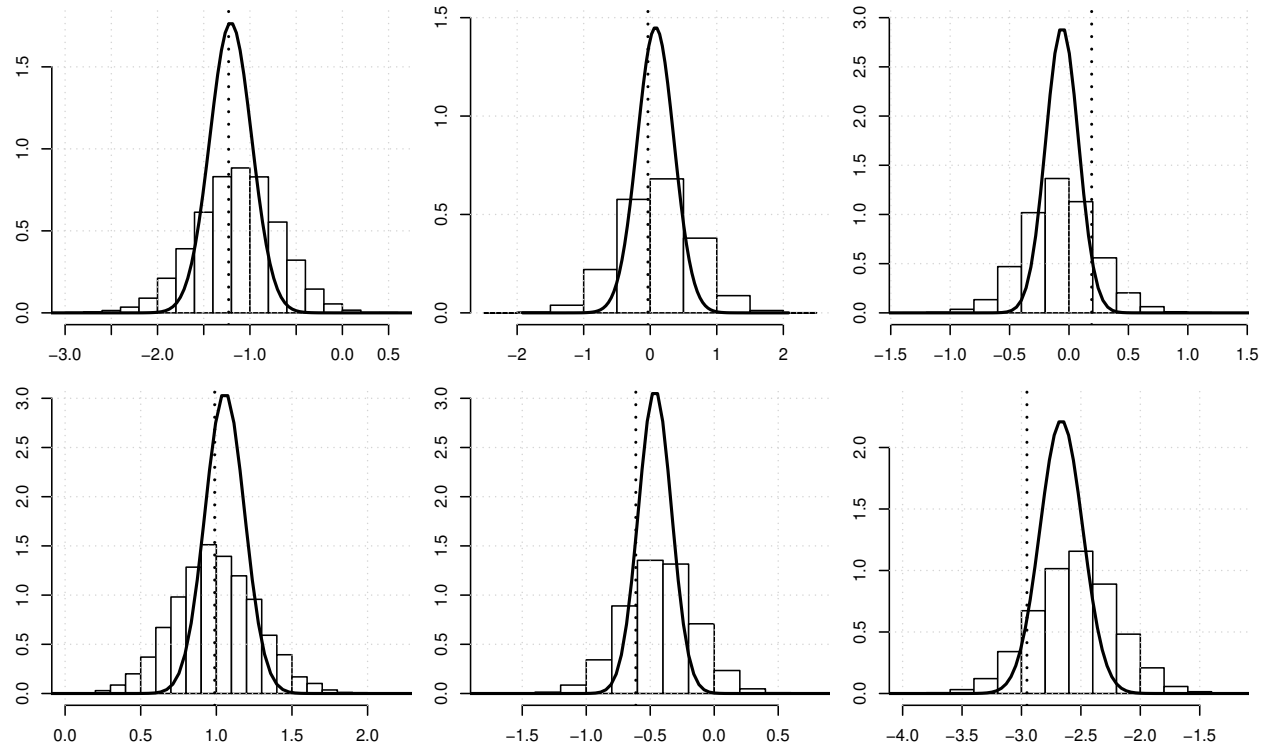

Figure 1. Plot to compare the limiting Gaussian distributions of the de-biased estimator and the histograms of the 10000 posterior samples for some entries. The dotted line is the true value of the entries. 


\section{References}

1. Alquier, P. (2013). Bayesian methods for low-rank matrix estimation: short survey and theoretical study. In Algorithmic Learning Theory 2013, pages 309-323. Springer.

2. Alquier, P., Cottet, V., Chopin, N., and Rousseau, J. (2014). Bayesian matrix completion: prior specification and consistency. arXiv preprint arXiv:1406.1440.

3. Alquier, P. and Ridgway, J. (2020). Concentration of tempered posteriors and of their variational approximations. The Annals of Statistics, 48(3):1475-1497.

4. Babacan, S. D., Luessi, M., Molina, R., and Katsaggelos, A. K. (2012). Sparse bayesian methods for low-rank matrix estimation. IEEE Transactions on Signal Processing, 60(8):3964-3977.

5. Bennett, J. and Lanning, S. (2007). The netflix prize. In Proceedings of KDD cup and workshop, volume 2007, page 35.

6. Candès, E. J. and Plan, Y. (2010). Matrix completion with noise. Proceedings of the IEEE, 98(6):925-936.

7. Candès, E. J. and Recht, B. (2009). Exact matrix completion via convex optimization. Found. Comput. Math., 9(6):717-772.

8. Candès, E. J. and Tao, T. (2010). The power of convex relaxation: near-optimal matrix completion. IEEE Trans. Inform. Theory, 56(5):2053-2080.

9. Chen, Y., Fan, J., Ma, C., and Yan, Y. (2019). Inference and uncertainty quantification for noisy matrix completion. Proceedings of the National Academy of Sciences, 116(46):22931-22937.

10. Cottet, V. and Alquier, P. (2018). 1-bit matrix completion: Pac-bayesian analysis of a variational approximation. Machine Learning, 107(3):579-603.

11. Hastie, T., Mazumder, R., Lee, J. D., and Zadeh, R. (2015). Matrix completion and low-rank svd via fast alternating least squares. The Journal of Machine Learning Research, 16(1):3367-3402.

12. Klopp, O. et al. (2015). Matrix completion by singular value thresholding: sharp bounds. Electronic journal of statistics, 9(2):2348-2369.

13. Koltchinskii, V., Lounici, K., and Tsybakov, A. B. (2011). Nuclear-norm penalization and optimal rates for noisy low-rank matrix completion. Ann. Statist., 39(5):2302-2329.

14. Kruschke, J. (2014). Doing Bayesian data analysis: A tutorial with R, JAGS, and Stan. Academic Press.

15. Lawrence, N. D. and Urtasun, R. (2009). Non-linear matrix factorization with gaussian processes. In Proceedings of the 26th Annual International Conference on Machine Learning, pages 601-608. ACM.

16. Lim, Y. J. and Teh, Y. W. (2007). Variational bayesian approach to movie rating prediction. In Proceedings of KDD Cup and Workshop, volume 7, pages 15-21.

17. Mai, T. T. and Alquier, P. (2015). A bayesian approach for noisy matrix completion: Optimal rate under general sampling distribution. Electron. J. Statist., 9(1):823-841.

18. Mazumder, R., Hastie, T., and Tibshirani, R. (2010). Spectral regularization algorithms for learning large incomplete matrices. The Journal of Machine Learning Research, 11:2287-2322.

19. McElreath, R. (2020). Statistical rethinking: A Bayesian course with examples in $R$ and Stan. CRC press.

20. Negahban, S. and Wainwright, M. J. (2012). Restricted strong convexity and weighted matrix completion: optimal bounds with noise. J. Mach. Learn. Res., 13:1665-1697.

21. Recht, B. and Ré, C. (2013). Parallel stochastic gradient algorithms for large-scale matrix completion. Math. Program. Comput., 5(2):201-226.

22. Salakhutdinov, R. and Mnih, A. (2008). Bayesian probabilistic matrix factorization using markov chain monte carlo. In Proceedings of the 25th international conference on Machine learning, pages 880-887. ACM.

23. Yang, L., Fang, J., Duan, H., Li, H., and Zeng, B. (2018). Fast low-rank bayesian matrix completion with hierarchical gaussian prior models. IEEE Transactions on Signal Processing, 66(11):2804-2817.

24. Zhou, M., Wang, C., Chen, M., Paisley, J., Dunson, D., and Carin, L. (2010). Nonparametric bayesian matrix completion. Proc. IEEE SAM. 